Recommendations The government to upgrade and accredit prison health units to offer comprehensive HIV/AIDS services Policies that enforce the rights of inmates to health services should be rejuvenated. Increase funding to improve service delivery.

Conclusion HIV/AIDS prevention can be scaled up among the inmates.

\section{P3.446 EXCELLENT EFFICACY OF PRITELIVIR (AIC316) IN SUPPRESSION OF GENITAL HERPES, A NOVEL DRUG AGAINST HERPES SIMPLEX VIRUS (HSV) TYPE 1 AND 2}

doi:10.1136/sextrans-2013-051184.0896

'A Birkmann, 'D McCormick, 'D Kropeit, 'B Timmler, 'S Stoelben, ${ }^{2} \mathrm{~A}$ Wald, ${ }^{3} \mathrm{H}$ Field, 'M P Richard, 'H Zimmermann, 'H Rübsamen-Schaeff. 'AiCuris GmbH \& Co. KG, Wuppertal, Germany; ${ }^{2}$ University of Washington, Seattle, WA, United States; ${ }^{3}$ University of Cambridge, Cambridge, UK

Genital Herpes is generally being caused by HSV-2, although genital infections with the "labial" herpes virus, HSV-1, have been increasing. Transmission of the herpes virus has become a major health concern since it also promotes transmission of other sexually transmitted diseases, e.g. HIV. Nucleoside analogues are widely used for treatment of genital herpes, but recurrences still occur after cessation of therapy and even under long-term treatment. Furthermore, genital transmission of HSV-2 cannot efficiently be prevented by existing drugs.

PRITELIVIR (AIC316) belongs to a novel class of antiviral compounds with activity against HSV-1 and HSV-2. In contrast to nucleoside analogues targeting the viral DNA polymerase PRITELIVIR (AIC316) prevents the de novo synthesis of viral DNA through inhibition of the viral helicase-primase complex. PRITELIVIR (AIC316) therefore does not require activation by the viral thymidine kinase and is protective for uninfected cells.

In vitro and in vivo, PRITELIVIR (AIC316) exhibited potent and rapid antiviral activity as well as superior efficacy against both HSV-1 and -2 compared to nucleoside analogues. In single and multiple dose phase I trials PRITELIVIR (AIC316) was safe with favourable pharmacokinetics resulting in a long half-life, indicative of efficacy with once per day dosing. In a recently completed phase II proof-of-concept and dose finding trial in subjects with genital herpes PRITELIVIR (AIC316) met the primary and secondary endpoint of the trial demonstrating excellent activity in reducing viral shedding in a dose dependent way and suppression of clinical symptoms, even with once weekly dosing. A head-to-head comparison trial with Valtrex is underway.

In conclusion, PRITELIVIR (AIC316) represents a highly active and novel treatment option for HSV-1 and -2 infections with very convenient dosing.

\section{P3.447 LEUKOCYTE EXTRACT MODULATES CELLULAR FACTORS INVOLVED IN HIV INFECTION}

doi:10.1136/sextrans-2013-051184.0897

'C B Fernandez-Ortega, 'D Casillas, ${ }^{2} \mathrm{M}$ Dubed, ${ }^{2} \mathrm{~L}$ Navea, ${ }^{1} \mathrm{~A}$ C Ramirez, 'L Lopez, 'T Paneque. 'Center for Genetic Engineering and Biotechnology, Havana, Cuba; ${ }^{2}$ AIDS Research Institute, San Jose, Cuba

Background Despite the therapeutic advances of antiretroviral, problems of drug resistance, latent viral reservoirs, and drug induced toxic effects that compromise effective viral control point to the need for new classes of anti-HIV drugs with different modes of action. An extract from human leukocyte called Dialyzable Leukocyte Extract (DLE) delays AIDS progression. However, the molecular bases supporting this effect are not completely comprehended. We hypothesises that active molecules from DLE modulate cellular factors involved in HIV infection.
Methods Using an in vitro infection model on MT4 cell line we demonstrated an inhibitory effect of DLE on HIV replication in treated cells which led us to identify molecules that could mediate this and other effects observed in this preparation.

Results DLE shows a significant inhibitory effect on HIV replication ranged from $80-90 \%$ according to the viral challenge. In addition, DLE shows modulation of important endogenous factors involved in HIV immunopathogenesis like TNFalpha and transcription factors NFkB and Sp1.

Conclusions DLE effect on cellular factors involved in HIV replication correlates with DLE inhibitory effect on HIV in vitro replication. The inhibition of HIV replication observed with DLE treatment could be mediated by TNF and transcriptions factors modulation involved in HIV infection. These finding could support the use of DLE on HIV patients and/or in health individuals to prevent the infection.

\section{P3.448 HPV AND ANOGENITAL WARTS. NEW ORIGINAL TREATMENT WITH ANALYSE AND OVERVIEW OF THE CURRENT CLINICAL SITUATION TODAY}

doi:10.1136/sextrans-2013-051184.0898

\section{E G Duray. Derma-Med Clinic, Budapest, Hungary}

Background HPV induced anogenital infections are the most common STI-s worldwide. According to WHO published data the incidence of them has dramatically raised in the past decade. Besides the high contagiosity benign epithelial lesions of the genitoanal region, the anogenital warts - HPV-s are responsible for development of most of the cervical neoplasias and other malignancies of the epithelial areas. Prevalence of HPV induced genital warts in the general population is $10 \%$ and affects $30 \%$ of sexually active young women at least $1 \mathrm{x}$ in their life. $65 \%$ of sexual partners of patients having condylomas will develop identical lesions in 9 months time. For the last decade no innovation has been proposed for treatment of anogenital warts. Currently available methods are based on surgical, chemical or immunological approaches.

Methods We have examined the efficacy and tolerability of an innovative cytotoxic method in the treatment of genitoanal condylomas in an international open prospective study. The cytodestructive effect is based on an innovative acid redox system (NO2-).

Results We have histological, clinical and virological evidence of the action of this system. The product is applied topically, it acts only locally, it doesn't destroy the basal membrane and induces mummification of the condyloma tissue. We have demonstrated by histological examination the similar activity of this procedure versus cryotherapy with the advantage of easier application, it is painless and doesn't need local anaesthesia.

Conclusion The treatment has great advantage in recurrent cases of genital warts by avoiding the need of repeated surgical procedures. It is also applicable for pregnant women, it doesn't need specific technical facilities and experience. This method has direct effect on HPV DNA.

\section{P3.449* THE PROMISE OF ESTRIOL CREAM FOR PREVENTION OF VAGINAL INFECTION IN PREMENOPAUSAL WOMEN}

doi:10.1136/sextrans-2013-051184.0899

${ }^{1}$ I. G. Dzuba, ${ }^{2}$ H. Jones, ${ }^{3} \mathrm{~S}$. A. Ballagh, ${ }^{4}$ W. H. Clark, ${ }^{5}$ S. Hillier, ${ }^{6}$ T. Irwin, ${ }^{7 P}$. Marx, ${ }^{8} \mathrm{~S}$ Smith, ${ }^{9}$ R. S. Veazey, 'B. Winikoff. 'Gynuity Health Projects, New York, NY, United States, ${ }^{2}$ CUNY School of Public Health at Hunter College, New York, NY, United States, ${ }^{3}$ Los Angeles County Harbor-UCLA Medical Center, Torrance, CA, United States, ${ }^{4}$ New York Presbyterian Hospital, New York, NY, United States, ${ }^{5}$ Magee-Womens Research Institute, Pittsburgh, PA, United States, ${ }^{6}$ University of Illinois at Chicago, Chicago, IL, United States, TTulane University School of Public Health and Tropical Medicine, New Orleans, LA United States, ${ }^{8}$ Smith Center for Infectious Diseases and Urban Health, East Orange, NJ, United States, ${ }^{9}$ Tulane National Primate Research Center, Covington, LA, United States. 